\title{
Experimental Demonstration of Widely Tunable Rate/Reach Adaptation From 80 km to 12,000 km Using Probabilistic Constellation Shaping
}

\author{
Joan M. Gené(1,2), Xi Chen ${ }^{(1)}$, Junho Cho ${ }^{(1)}$, Sethumadhavan Chandrasekhar ${ }^{(1)}$ and Peter Winzer $^{(1)}$ \\ (1) Nokia Bell Labs, 791 Holmdel Road, Holmdel 07733, NJ, USA. \\ (2) Universitat Politècnica de Cataluya, Jordi Girona, 1-3, Barcelona, 08034, Spain. \\ joan.gene@upc.edu,xi.v.chen@nokia-bell-labs.com,junho.cho@nokia-bell-labs.com,s.chandrasekhar@ieee.org. \\ peter.winzer@nokia-bell-labs.com
}

\begin{abstract}
We experimentally demonstrate the rate/reach adaptability of probabilistically constellation-shaped quadrature amplitude modulation across from $80 \mathrm{~km}$ to $12,000 \mathrm{~km}$ using the same $32-$ GBaud transponder hardware and highlight the roles of template and shaping distribution.
\end{abstract}

\section{Introduction}

Probabilistic constellation shaping (PCS) has become a key ingredient to modern optical transponders [1], allowing both a performance improvement compared to square quadrature amplitude modulation (QAM) as well as a finegrained software-defined trade-off between the transponder's achievable interface rate, spectral efficiency, and transmission distance [2]-[5]. Record research experiments using PCS have targeted widely different applications, from datacenter interconnects (DCIs) [6] to terrestrial long-haul [3], [7] and submarine [4], [8] transmission, have reached net interface rates as high as $1.3 \mathrm{~Tb} / \mathrm{s}$ per carrier [9], and have used constellation templates as high as 16,384-QAM [10]. These (and other similar) experiments have each set records across fairly small ranges of transmission reach, but the wide tunability of PCS across the full distance range from DCI to trans-Pacific transmission has not yet been experimentally demonstrated on a single hardware platform. Here, we report transmission results from $80 \mathrm{~km}$ to 12,000 $\mathrm{km}$ using a common transponder hardware platform at 32 GBaud, using 16, 64, 256 and 1024-ary QAM templates.

\section{Experimental Setup}

The experimental setup is shown in Fig. 1. At the transmitter (TX), an external cavity laser (ECL) at $1550.08 \mathrm{~nm}$, with a linewidth of $1 \mathrm{kHz}$, is modulated at $32 \mathrm{GBaud}$ using a lithium niobate in-phase/quadrature modulator (IQM; $35 \mathrm{GHz}$ 3-dB bandwidth and $\mathrm{V}_{\pi}=3.5 \mathrm{~V}$ ), driven by 2 outputs of an 8-bit, 4-channel, CMOS-based 88-GSa/s digital-to-analog converter (DAC) generating a random stream of 95,325 symbols. A fiber-delay decorrelator (10.9 m; 1,744 symbols) is used to emulate polarization-division multiplexing (PDM). Erbium-doped fiber amplifiers (EDFAs) compensate for TX losses. The transmission link is implemented as a $400-\mathrm{km}$ long recirculating loop, consisting of five $80-\mathrm{km}$ spans of standard single-mode fiber (SSMF). EDFAs are used to compensate for span losses, and optical filters (OFs) following each EDFA reduce the build-up of amplified spontaneous emission (ASE). The per-span input powers are set by variable optical attenuators (VOAs). A 150-GHz-bandwidth wavelength-selective switch (WSS) is included to further eliminate out-of-band ASE. At the receiver (RX), another 100-GHz-bandwidth WSS pre-filters the signal, and detection is done using an intradyne coherent receiver consisting of two $90^{\circ}$ hybrids, four balanced photodetectors (BPDs, $45-\mathrm{GHz} 3-\mathrm{dB}$ bandwidth) and a 4-channel real-time oscilloscope operating at $256 \mathrm{GSa} / \mathrm{s}$ with an analog bandwidth of $103 \mathrm{GHz}$ and a nominal resolution of 10 bits. The local oscillator (LO) has the same specifications as the transmission laser and is tuned to within a $2.5-\mathrm{GHz}$ frequency offset from the signal.

The signal constellations are generated using probabilistic amplitude shaping (PAS) [2] based on Maxwell-Boltzmann (MB) amplitude distributions using the parameters summarized in Tab. 1, taking into account PDM; $M$-ary QAM templates with $M=16,64,256,1024$ generate PCS-M-QAM. (At $3,200 \mathrm{~km}$, we also test constellations with
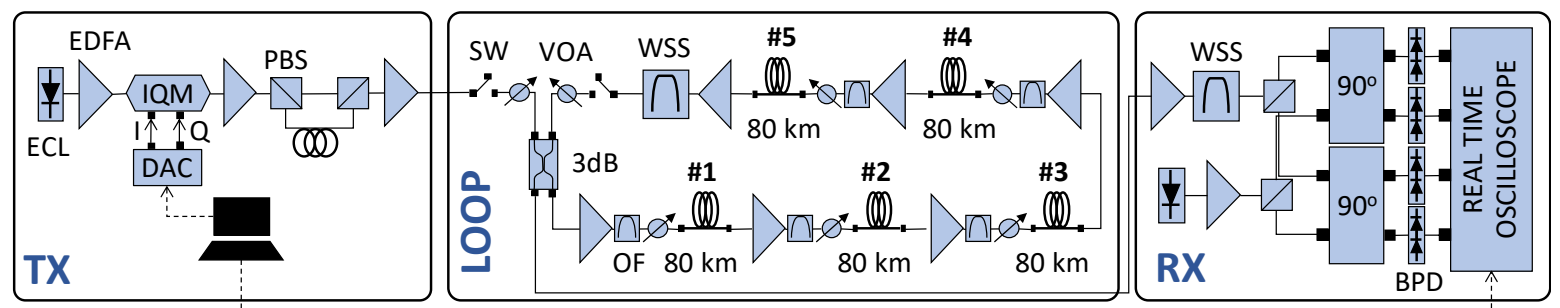

Fig. 1. Experimental Setup. 
Table1. PCS-M-QAM (dual-polarization) parameters.

\begin{tabular}{|r|c|c|c|c|}
\hline$M=2^{m}$ & 16 & 64 & 256 & 1024 \\
\hline SC-LDPC Code Rate & $3 / 4$ & $3 / 4$ & $4 / 5$ & $5 / 6$ \\
\hline HD-BCH Code Rate & 0.9915 & 0.9915 & 0.9915 & 0.9915 \\
\hline Concatenated FEC Rate $\left(R_{c}\right)$ & 0.7436 & 0.7436 & 0.7932 & 0.8262 \\
\hline $\mathrm{NGMI}_{\text {th }}$ & 0.8105 & 0.8105 & 0.8456 & 0.8714 \\
\hline $\mathrm{IR}=4 \cdot\left(\beta+1-\left(1-\mathrm{R}_{\mathrm{c}}\right) \cdot \mathrm{m} / 2\right)$ & $4 \cdot(\beta+0.487)$ & $4 \cdot(\beta+0.231)$ & $4 \cdot(\beta+0.173)$ & $4 \cdot(\beta+0.131)$ \\
\hline$\beta_{\max }=\mathrm{m} / 2-1$ & 1 & 2 & 3 & 4 \\
\hline $\mathrm{IR}_{\max }$ & 5.95 & 8.92 & 12.69 & 16.52 \\
\hline
\end{tabular}

generalized MB (GMB) distributions, adjustable to be more tolerant to Kerr nonlinearity than MB distributions, as discussed below.) A 1\% root-raised-cosine roll-off is used. For forward-error correction (FEC), soft-decision (SD) spatially-coupled low-density parity-check (SC-LDPC) codes of three different code rates are used as an inner code, followed by a rate- 0.9915 hard-decision Bose-Chaudhuri-Hocquenghem (HD-BCH) code to remove potential error floors. For each concatenated code of Tab. 1, the normalized generalized mutual information (NGMI) threshold NGMI determined in an auxiliary additive white Gaussian noise channel. This threshold has been shown to reliably predict decoding performance for PCS [11], and is used to determine the maximum viable shaping parameter $\beta$, yielding a maximum viable information rate (IR); $\beta_{\max }$ and $\mathrm{IR}_{\max }$ refer to the highest possible $\beta$ and IR for each $M$, yielding unshaped uniform QAM.

\section{Results and Discussion}

To find the maximum viable IR under our system assumptions for a particular $M$ at a given distance, we first sweep the signal launch power using the expected optimum value of $\beta$, cf. Fig. 2(a). (For a given distance, the optimum launch power depends only mildly on $\beta$.) At the identified optimum launch power, we then vary $\beta$ in fine steps and measure the NGMI to determine the maximum viable $\beta$ at NGMI $_{\text {th }}$ cf. Fig. 2(b). The high accuracy of the measurements is evident from the small error bars (mean \pm std. dev.) within a set of 20 measurements per point.

Figure 3a shows the measured IRs from $80 \mathrm{~km}$ to $12,000 \mathrm{~km}$ for the 4 different QAM templates as well as the Shannon limit (solid black line) for our system including ASE and nonlinear interference noise [12], revealing an implementation penalty of $\sim 4.5 \mathrm{~dB}$ for our transponder. The maximum possible IRs for each QAM template (IR ${ }_{\max }$ ) are shown as dotted horizontal lines. Importantly, note from the crossing of the curves for different $M$ as highlighted in the inset that the largest possible QAM template is not the best one to choose, as larger templates degrade PCS performance due to the enhanced visibility of FEC coding gaps [13]. We find that the optimum template for a given $\beta$ is the one that has the smallest $\beta_{\max }$ satisfying $\beta_{\max }-\gamma /\left(1+\beta_{\max }\right)>\beta$, with an implementation-specific value of $\gamma$ which is 0.73 for our system; At $12,000 \mathrm{~km}$, PCS based on a 1024-QAM template is $\sim 0.5 \mathrm{~b} / \mathrm{s} / \mathrm{Hz}$ worse than PCS based on a 16-QAM template. According to our measurements, the optimum QAM templates are: 1024-QAM up to $\sim 600 \mathrm{~km} ; 256-\mathrm{QAM}$ up to $\sim 1,500 \mathrm{~km}, 64-\mathrm{QAM}$ up to $\sim 4,500 \mathrm{~km}$, and 16-QAM for longer distances. With the best configuration at each distance, the IRs draw an the almost straight dotted black line. Every doubling in distance reduces the IR by $\sim 2 \mathrm{bit} / \mathrm{symbol}$, corresponding to a 6-dB effective SNR drop. The IR can be continuously varied between $\sim 15.68 \mathrm{bit} / \mathrm{symbol}$ at $80 \mathrm{~km}$ and $\sim 2.83 \mathrm{bit} / \mathrm{symbol}$ at $12,000 \mathrm{~km}$. As can be seen in Fig. 3a, PCS-1024-QAM at $80 \mathrm{~km}$ still has a gap of $\sim 0.8 \mathrm{bit} / \mathrm{symbol}$ to its $\mathrm{IR}_{\max }$.
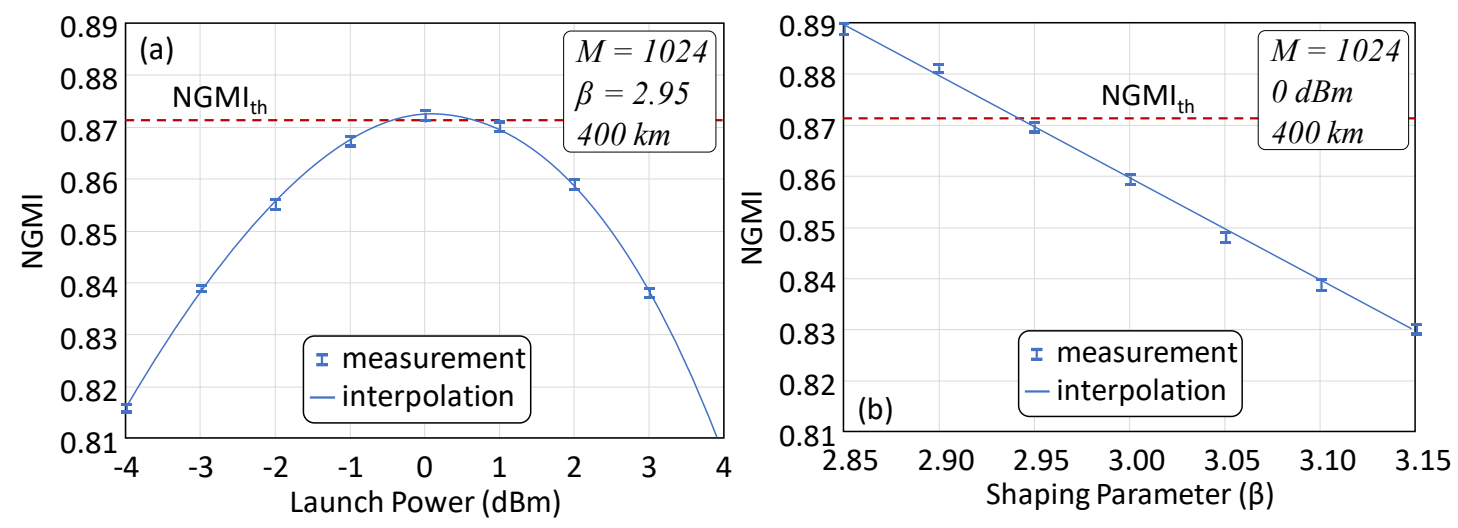

Fig. 2. (a) Launch power sweep and cubic interpolation. (b) Shaping parameter sweep and linear fit. 

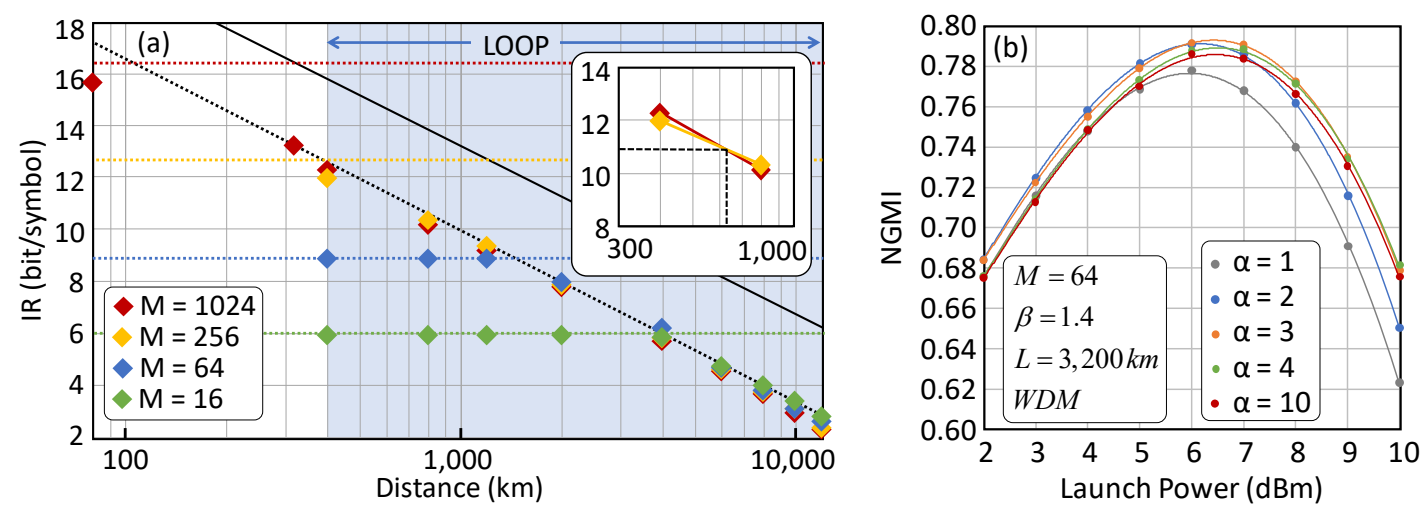

Fig. 3. (a) IR vs. distance. (b) NGMI vs. optical launch power using GMB distributions with different exponents $(\alpha)$.

At $3,200 \mathrm{~km}$, we also tested GMB distributions whose probability at amplitude $x$ is given by $P(x)=$ $\exp \left(-\lambda x^{\alpha}\right) / \sum_{x^{\prime} \in X} \exp \left(-\lambda x^{\prime \alpha}\right)$, where $\mathcal{X}$ denotes the set of QAM amplitudes and $\lambda$ determines the entropy (i.e., the shaping parameter $\beta$ for an ideal shaping algorithm). An MB distribution is a special case of the GMB distributions with $\alpha=2$. For a fixed $\beta$, the GMB distribution becomes flatter near zero amplitude (i.e., more uniform-like) as $\alpha$ increases; therefore, a larger $\alpha$ decreases the kurtosis that causes severer Kerr nonlinearities [14], at the expense of a reduced linear shaping gain at the same time. In order to emphasize nonlinear effects, we implemented a 5-channel WDM transmission experiment (50 GHz channel spacing). Figure $3 \mathrm{~b}$ shows the measured NGMI vs. optical launch power for $\alpha=1,2,3,4,10$, which is in agreement with theory [14], showing that a greater $\alpha$ enhances the nonlinear tolerance in the nonlinear (high-power) regime, while it generally incurs a larger shaping gap in the linear (low-power) regime. However, the interplay of linear and nonlinear effects of the GMB does not yield a noticeably better peak performance compared to the MB distribution $(\alpha=2)$; this is in contradiction to the experimental demonstration in Ref. [15] that observed $0.25-\mathrm{dB}$ gain in the $\mathrm{Q}$ factor in a similar experimental condition.

\section{Conclusion}

We experimentally demonstrated PCS transmission over a wide range of transmission distances, from $80 \mathrm{~km}$ to 12,000 $\mathrm{km}$ using a single transponder hardware platform. We showed how the best QAM template is found and find that nonMB distributions do not yield a substantial benefit at the optimum launch power.

\section{References}

[1] "Nokia Corporation, Nokia Photonic Service Engine 3," 2018. [Online]. Available: https://networks.nokia.com/photonic-service-engine-3.

[2] G. Bocherer, F. Steiner, and P. Schulte, "Bandwidth Efficient and Rate-Matched Low-Density Parity-Check Coded Modulation," IEEE Trans. Commun., vol. 63, no. 12, pp. 4651-4665, Dec. 2015.

[3] F. Buchali, F. Steiner, G. Bocherer, L. Schmalen, P. Schulte, and W. Idler, "Rate Adaptation and Reach Increase by Probabilistically Shaped 64-QAM: An Experimental Demonstration,” J. Light. Technol., vol. 34, no. 7, pp. 1599-1609, Apr. 2016.

[4] A. Ghazisaeidi et al., "65Tb/s Transoceanic Transmission Using Probabilistically-Shaped PDM-64QAM," in 42nd European Conference on Optical Communication (ECOC), 2016, p. PD Paper Th.3.C.4.

[5] J. Cho and P. J. Winzer, "Probabilistic Constellation Shaping for Optical Fiber Communications," J. Light. Technol., vol. 37, no. 6, pp. 15901607, Mar. 2019.

[6] S. L. I. Olsson, J. Cho, S. Chandrasekhar, X. Chen, P. J. Winzer, and S. Makovejs, "Probabilistically shaped PDM 4096-QAM transmission over up to $200 \mathrm{~km}$ of fiber using standard intradyne detection," Opt. Express, vol. 26, no. 4, p. 4522, 2018.

[7] S. Chandrasekhar et al., "High-spectral-efficiency transmission of PDM 256-QAM with Parallel Probabilistic Shaping at Record Rate-Reach Trade-offs," in ECOC 2016 - Post Deadline Paper; 42nd European Conference on Optical Communication, 2016 , pp. 1-3.

[8] J. Cho et al., "Trans-Atlantic Field Trial Using High Spectral Efficiency Probabilistically Shaped 64-QAM and Single-Carrier Real-Time 250-Gb/s 16-QAM,” J. Light. Technol., vol. 36, no. 1, pp. 103-113, Jan. 2018.

[9] F. Buchali et al., "1.3-Tb/s Single-Channel and 50.8-Tb/s WDM Transmission Over Field-Deployed Fiber," in European Conference on Optical Communications (ECOC), 2019, p. PD1.3.

[10] X. Chen, J. Cho, A. Adamiecki, and P. Winzer, “16384-QAM Transmission at 10 GBd Over 25-Km SSMF Using Polarization-Multiplexed Probabilistic Constellation Shaping," in European Conference on Optical Communications (ECOC), 2019, p. PD3.3.

[11] J. Cho, X. Chen, S. Chandrasekhar, and P. Winzer, "On Line Rates, Information Rates, and Spectral Efficiencies in Probabilistically Shaped QAM systems," Opt. Express, vol. 26, no. 8, p. 9784, 2018.

[12] P. Poggiolini et al., "The GN-model of Fiber Non-linear Propagation and its Applications,” J. Light. Tech., vol. 32 , no. 4 , pp. 694, Feb. 2014.

[13] J. Cho, S. L. I. Olsson, S. Chandrasekhar, and P. Winzer, "Information Rate of Probabilistically Shaped QAM with Non-Ideal Forward Error Correction," in European Conference on Optical Communication, ECOC, 2018, vol. 2018-September.

[14] T. Fehenberger, A. Alvarado, G. Bocherer, and N. Hanik, "On Probabilistic Shaping of Quadrature Amplitude Modulation for the Nonlinear Fiber Channel," J. Light. Technol., vol. 34, no. 21, pp. 5063-5073, 2016.

[15] M. N. Tehrani, M. Torbatian, H. Sun, P. Mertz, and K. T. Wu, "A Novel Nonlinearity Tolerant Super-Gaussian Distribution for Probabilistically Shaped Modulation,” in European Conference on Optical Communication, ECOC, 2018, vol. 2018-September. 